\title{
Synergistic Effects of Mixed Silica Micro-nanoparticles on Compressive Dynamic Stiffness and Damping of Epoxy Adhesive
}

\author{
Yohanes $^{1}$ (D) . Y. Sekiguchi ${ }^{1}$
}

Received: 20 December 2017 / Accepted: 5 March 2018 / Published online: 15 March 2018

(C) The Author(s) 2018

\begin{abstract}
Dynamic stiffness and damping of epoxy adhesives are critical for ensuring the safety, reliability, and comfort of structures subjected to vibrations and impact loads. This study conducts split Hopkinson pressure bar (SHPB) tests to investigate the synergistic effects of silica micro-nanoparticles on these critical properties. Micro-nanoparticle content and composition ratio purity are varied at 2,5 , and $10 \%$ by weight (wt $\%$ ) and from $0 \%$ (pure microparticles) to $100 \%$ (pure nanoparticles), respectively. Positive simultaneous stiffening and energy absorption effects are observed at a silica content of $5 \mathrm{wt} \%$ owing to improved nanoparticle dispersion; this increases the interface area and induces cooperative matrix-filler interactions. At this silica content and a composition ratio of 50\%, stiffness and damping are 45 and $40 \%$ larger than those of neat epoxy, respectively. Silica micro-nanoparticles are less effective in improving particle dispersion at more than $5 \mathrm{wt} \%$. Conventional mechanical dispersion is limited to applications below a certain silica content; the results suggest a simple, low-cost dispersion technique as an alternative to the in-situ technique and provide options for designing epoxy stiffness and damping appropriate for specific applications.
\end{abstract}

Keywords Dynamic stiffness $\cdot$ Hysteretic damping $\cdot$ Epoxy $\cdot$ Silica filler $\cdot$ Split Hopkinson pressure bar

\section{Introduction}

In lightweight structural applications such as spacecraft and automobile parts, epoxy-based adhesives and composites are superior to metal owing to their high strength-to-weight ratio and strong adhesion to different materials. Structures subjected to dynamic loads such as vibrations and impacts must be well-designed to ensure their safety, reliability, and comfort. Therefore, dynamic stiffness and damping characterizations of epoxy are critical for structural design and analysis.

In practice, high-modulus silica particles are commonly used to stiffen and toughen epoxy adhesive by exploiting inter-particle interactions, matrix-filler interactions, and the inherent properties of silica particles in a matrix. Welldispersed silica nanoparticles stiffen and toughen an epoxy adhesive more efficiently than silica microparticles owing

Yohanes

d146781@hiroshima-u.ac.jp

Y. Sekiguchi

sekiguch@hiroshima-u.ac.jp

1 Graduate School of Engineering, Institute of Engineering, Hiroshima University, Higashihiroshima, Japan to their larger surface-to-volume ratio, forming a more substantial matrix-filler interface area at a given weight fraction [1-7]. However, without any treatment, high-silica-content nanoparticles are difficult to disperse uniformly using a conventional mechanical mixer and tend to aggregate/agglomerate in the epoxy matrix [8-10].

Composing an appropriate composition ratio between two sizes of silica particles for a given size ratio reduces viscosity and breaks up the agglomerate, thus improving particle dispersion [11-14]. Additionally, such bimodal silica particles induce more complex matrix-filler and inter-particle interactions, which generate concurring effects on epoxy dynamic properties [14-16]. Nevertheless, such deagglomeration effectivity over a wide range of silica weight fractions and its subsequent collaborative effects on epoxy dynamic stiffness and damping, especially at intermediate-strain-rate loading, have been given little attention.

Experimental work has been conducted to investigate the synergistic effects of two-size silica particles on epoxy stiffness and fracture energy absorption with respect to particle composition ratio. Kwon et al. [14, 15] and Dittanet and Pearson [16] found an appropriate composition ratio of two-size silica particles that cooperatively 
toughens epoxy and ascribed this to better particle dispersion. Shariati et al. [17] found good dispersion at $1.5-6$ wt\% for two-size silica nanoparticles of 17 and $65 \mathrm{~nm}$ diameters but did not find any considerable coactive effects on stiffness and fracture energy absorption. Such previous works have indicated that the interplay of particle content, size ratio, and composition ratio affect the particle distribution and subsequent collaborative effects, which is consistent with suggestions made by Greenwood et al. $[11,12]$ and Dames et al. [13]. It is important to note that these previous studies focused on epoxy behavior under static loading, which may differ from that under dynamic loading owing to strain rate sensitivity of the epoxy and of matrix-filler interactions.

The effects of silica particles on epoxy behavior at highstrain-rate loading have been investigated using split Hopkinson pressure bars (SHPB). Miao et al. [18] and Tian et al. [19] reported that silica nanoparticles stiffen and strengthen epoxy composites at both quasi-static and dynamic loading. Silica nanoparticles stiffen epoxy efficiently at low-strainrate loading [19]. Islam et al. [7] showed that dynamic stiffness increases with silica particle content dispersed in the epoxy matrix. However, such studies focused on single-size silica-filled epoxy dynamic behavior at high-strain-rate loading.

More recently, in the authors' previous paper [20], we estimated the elastic-dynamic stiffness and damping of epoxy filled with silica micro-nanoparticles under a strain rate loading of $140 \mathrm{~s}^{-1}$ using SHPB with sandwiched specimens. We observed that epoxy filled with two-size silica particles exhibited greater damping, but lower stiffness compared to that of single silica particles. However, the damping was not precisely quantified because of the limitation of the sandwiched SHPB.

In the present paper, the objective is to investigate the synergistic effects of silica micro-nanoparticles of varied composition ratio and weight fraction on epoxy dynamic stiffness and damping in the elastic region. The SHPB method with bonded specimens is used to generate a complete loading-unloading elastic stress-strain response for epoxy filled with silica micro-nanoparticles and to precisely estimate damping. The results in this paper fill a knowledge gap regarding epoxy/silica dynamic behavior between quasistatic and high-strain-rate loading.

The experimental evidence suggests that the deagglomeration effectivity and collaborative effects of silica micronanoparticles are limited by silica particle content and vary with the composition ratio. Silica micro-nanoparticles offer a simple and low-cost solution to the nanoparticle dispersion problem in conventional mechanical mixers; moreover, they provide options for designing epoxy dynamic stiffness and damping to cater for specific applications by varying the composition ratio.
This paper presents the SHPB method with bonded specimens, data validation, and dynamic stiffness and damping estimations. It also reports and discusses the effects of silica micro-nanoparticle weight fraction and composition ratio on epoxy dynamic stiffness and damping.

\section{Method}

Split Hopkinson pressure bars (SHPB) have been used to measure the dynamic stress-strain response of brittle materials $[21,22]$ and to characterize the elastic behavior of metals [23]. The dynamic stiffness is represented by Young's modulus, which is estimated from the initial stress-strain slope. Hysteretic damping, which represents the ability of a material to dissipate mechanical energy, is estimated from the area covered by a complete loading-unloading (closedloop) stress-strain curve.

In SHPB with sandwiched specimens [20], precise damping estimation is difficult owing to incomplete stress-strain responses, which are ascribed to poor bar-specimen contact during unloading. In the present work, epoxy/silica adhesive was bonded to both input and output bars to maintain bar-specimen contact and to generate closed-loop stress-strain responses.

\section{Materials and Specimen Preparation}

The specimens were made from two-parts epoxy brand Scotch-Weld 1838 (B/A) filled with silica micro-nanoparticles. The physical properties of epoxy and silica particles are given in Table 1. The weight fraction and composition ratio of dispersed silica particles were varied as shown in Table 2 . The specimens were prepared according to the following procedure to ensure consistency: first, silica micro-nanoparticles at the desired ratio were mixed with epoxy base (B) and accelerator (A) using a planetary centrifugal mixer and were then degassed. Second, the pressure bars' bonded areas were heated and the epoxy specimen of thickness and diameter equal to 5 and $20 \mathrm{~mm}$ were bonded between them. Finally, the specimens were cured at room temperature for $1 \mathrm{~h}$ and were then heated to $65^{\circ} \mathrm{C}$ for $3 \mathrm{~h}$. The specimen was cooled to room temperature for $24 \mathrm{~h}$ before testing.

Table 1 Physical properties of epoxy and silica particles

\begin{tabular}{llll}
\hline Physical parameter & Micro silica & Nano silica & Epoxy adhesive \\
\hline Weight density $\left(\mathrm{g} / \mathrm{cm}^{3}\right)$ & 2.65 & & 1.21 \\
$\begin{array}{l}\text { Average diameter } \\
\text { Specific surface area }\end{array}$ & $34 \mu \mathrm{m}$ & $17 \mathrm{~nm}$ & - \\
$\left(\mathrm{m}^{2} / \mathrm{g}\right)$ & & 80 & - \\
\hline
\end{tabular}


Table 2 Calculated surface area of pre-mixed silica micronanoparticles

\begin{tabular}{llllll}
\hline $\begin{array}{l}\text { Composition ratio } \\
{[(\phi) \%]}\end{array}$ & $\begin{array}{l}\text { Micro silica } \\
(\%)\end{array}$ & Nano silica $(\%)$ & \multicolumn{2}{l}{ Pre-mixed surface area $\left(\mathrm{m}^{2}\right)$} & \\
\cline { 4 - 6 } & & & $2 \mathrm{wt} \%$ & $5 \mathrm{wt} \%$ & $10 \mathrm{wt} \%$ \\
\hline 0 & 100 & 0 & $(1.01 \mathrm{vol} \%)$ & $(2.55 \mathrm{vol} \%)$ & $(5.24 \mathrm{vol} \%)$ \\
25 & 75 & 25 & 0.27 & 0.68 & 1.36 \\
50 & 50 & 50 & 1.80 & 4.51 & 9.02 \\
75 & 25 & 75 & 3.34 & 8.34 & 16.68 \\
100 & 0 & 100 & 4.87 & 12.17 & 24.34 \\
\hline
\end{tabular}

Bracketed values indicate the conversion of silica content from weight fraction to volume fraction (calculated)

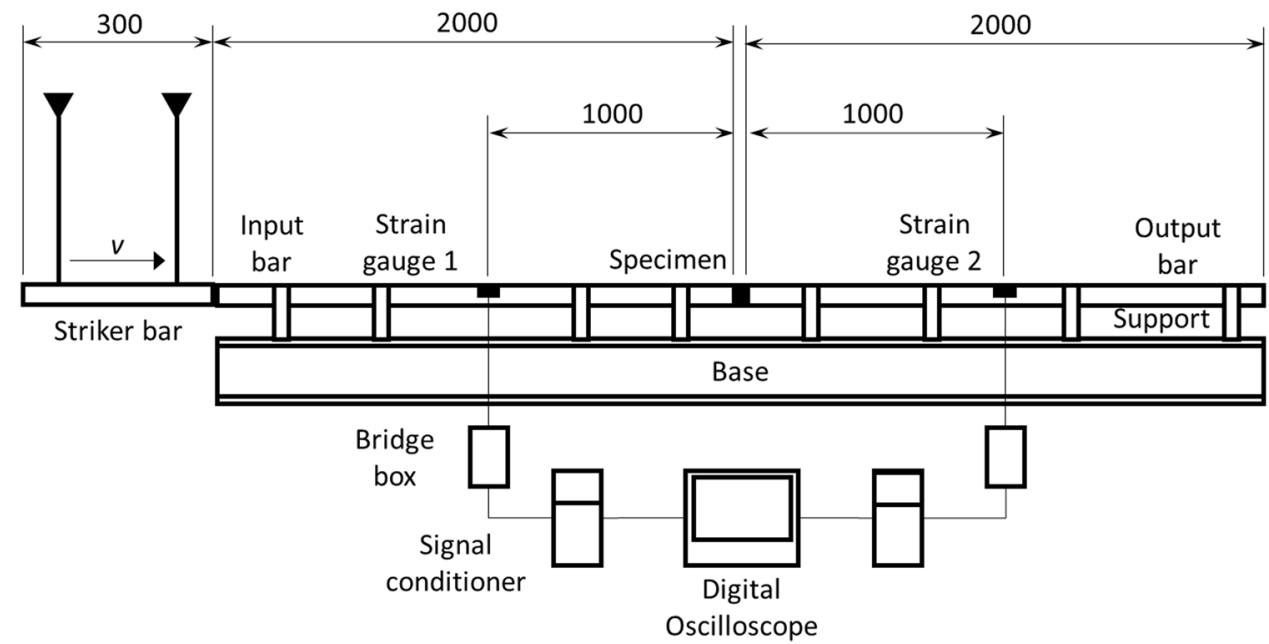

Fig. 1 Split Hopkinson pressure bar test apparatus with bonded specimen (units are in mm)

\section{SHPB Test Apparatus}

The SHPB used in the present work was composed of a striker bar, two pressure bars, and strain wave measurement instrumentation as shown in Fig. 1. All bars were made from SK-5 steel bars with a Young's modulus of $206 \mathrm{GPa}$ and diameters of $20 \mathrm{~mm}$. The impact side of the striker bar was slightly rounded to increase the rise time and avoid fluctuation in the early portion of the generated incident pulse. The appropriate length of the pulse rise time ensures dynamic stress equilibrium in the specimen while a smooth incident pulse avoids distortion in the early part of the stress-strain response. Both characteristics are critical in obtaining valid and accurate data in SHPB tests.

A compressive incident pulse $\left(\varepsilon_{I}\right)$ of $200 \mu$ s in loading duration was generated during the impact of the striker bar on the input bar. At the bar-specimen interface, some part of the incident pulse is reflected $\left(\varepsilon_{R}\right)$ to the input bar and the rest is transmitted $\left(\varepsilon_{T}\right)$ to the output bar. Strain gauges were positioned in the middle of both pressure bars to avoid signal overlapping so that complete strain loading and unloading could be recorded. Such complete strain history for the

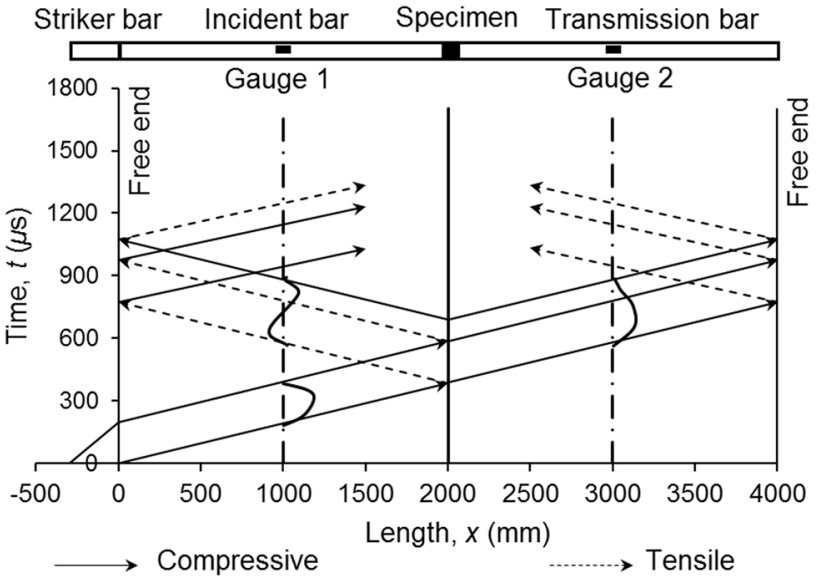

Fig. 2 Split Hopkinson bar arrangement and Lagrangian diagram showing gauge locations that avoid overlap of the measured strain waves

specimen resulted in a completely closed-loop stress-strain response, which is required to accurately estimate damping (Fig. 2). 


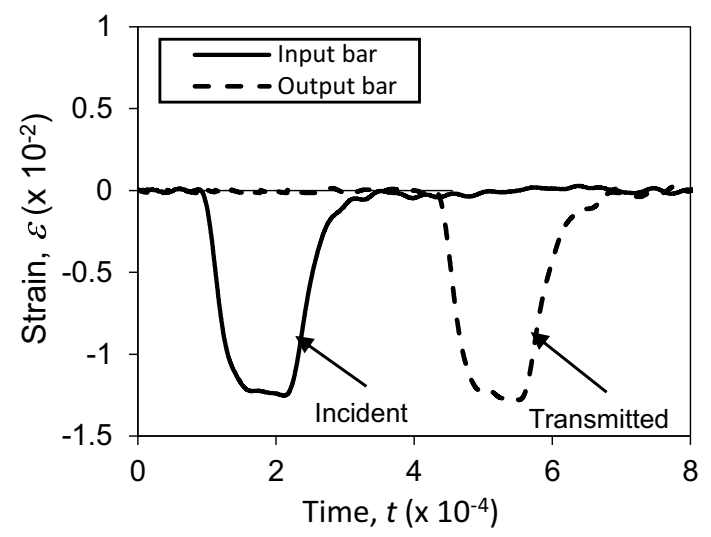

Fig. 3 Strain waves measured without specimen

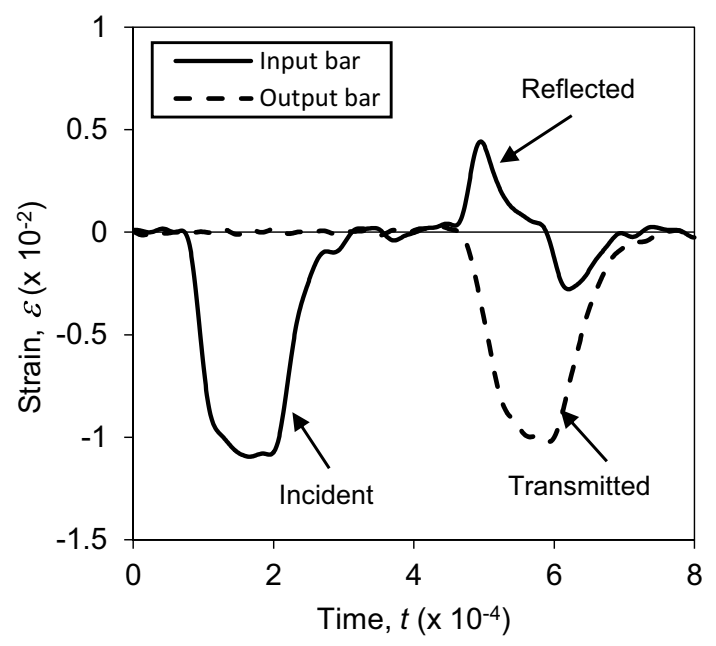

Fig. 4 Strain waves measured with specimen

\section{Test Calibration and Data Validity}

A calibration test was conducted on pressure bars without specimens to ensure the validity and accuracy of the obtained data. Identical incident and transmitted pulses obtained from measurement of pressure bars without specimens (Fig. 3) indicated good alignment of the SHPB system and ensured that the reflected pulse measured in Fig. 4 was contributed only by the specimen.

Figure 4 shows that the crowned striker bar effectively generated a smooth incident pulse with appropriate rise time. This ensured that the dynamic equilibrium condition in the specimen during loading and unloading was achieved, as shown in Fig. 5. The forces at both sides of the specimens are formulated as:

$$
\begin{aligned}
& P_{1}=A_{b} E\left\{\varepsilon_{I}(t)+\varepsilon_{R}(t)\right\} \\
& P_{2}=A_{b} E \varepsilon_{T}(t)
\end{aligned}
$$

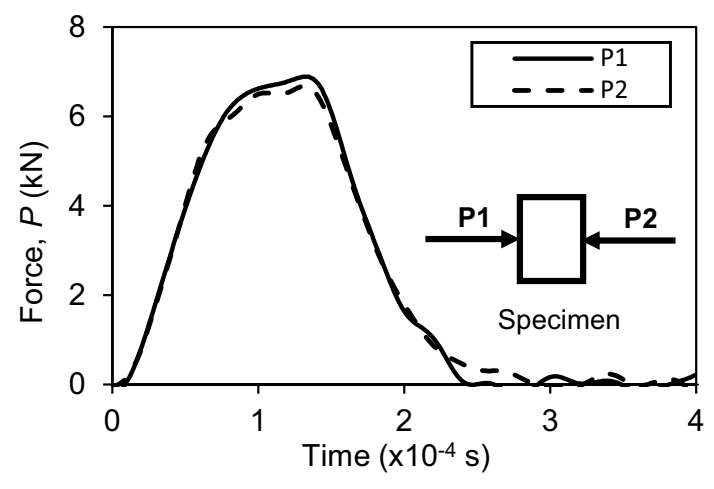

Fig. 5 Dynamic stress equilibrium on both sides of the specimen

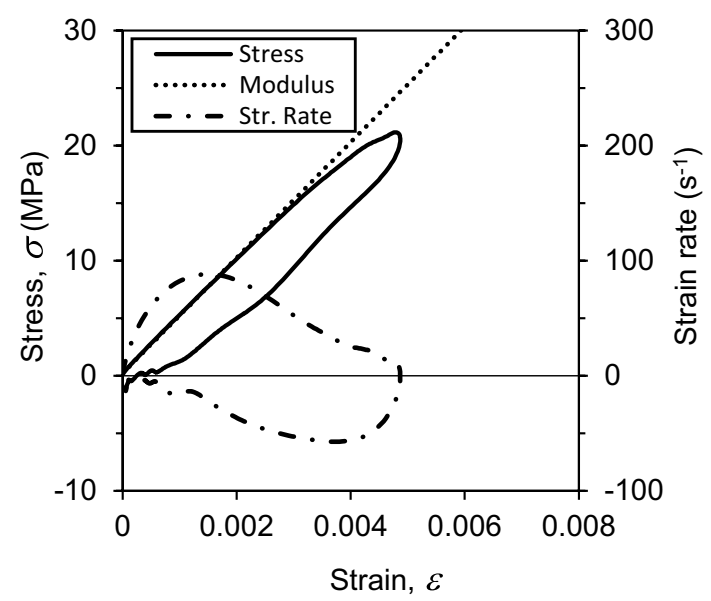

Fig. 6 Estimation of stiffness and damping from the slope and closedloop area of the stress-strain response. In the analysis, the strain rate is averaged from the loading part

where $A_{\mathrm{b}}$ is the pressure bars' cross-sectional area, $E$ is the Young's modulus of the bars, and $\varepsilon_{I}, \varepsilon_{R}$, and $\varepsilon_{T}$ are the aligned incident, reflected, and transmitted pulses, respectively.

The nearly constant strain rate during the test period were not obtained, as represented by the reflected wave in Fig. 4, owing to the limitation of the pulse-shaping technique used and the bonded constraints at the interface of specimen and pressure bars. However, such strain acceleration does not significantly affect the estimations and analysis of dynamic stiffness and damping in the present measurement condition.

\section{Estimation of Dynamic Stiffness and Hysteretic Damping}

Validated SHPB data were used to generate stress-strain responses (Fig. 6) for the specimens using the following equations:

$\sigma_{n}=E A_{b} / A_{s} \varepsilon_{T}(t)$ 
$\dot{\varepsilon}_{n}=2 C_{0} / L_{s} \varepsilon_{R}(t)$

$\varepsilon_{n}=2 C_{0} / L_{s} \int \varepsilon_{R}(t) d t$

where $C_{0}$ is the elastic wave speed through the bars, $L_{\mathrm{s}}$ is the specimen thickness, and $t$ is the time duration.

The stress-strain in the small strain range was nearly linear. Dynamic stiffness was estimated by fitting the slope of the line to the initial part of the stress-strain curve in Fig. 6. Hysteretic damping was estimated from the integral of the closed-loop area of the stress-strain curve using the following equation:

$\delta=\int \sigma(t) d \varepsilon(t)$

The reproducibility and consistency of measured data are indicated by the nearly overlapped stress-strain response of two specimens with a given silica weight fraction and composition ratio, as shown in Fig. 7.

\section{Results and Discussion}

Tests were conducted to investigate the effects of silica micro-nanoparticles on the dynamic compressive stiffness and damping of epoxy adhesive. A set of SHPBs with bonded specimens was used to generate stress-strain loops at the average strain rate of $80 \pm 10 \mathrm{~s}^{-1}$. Several tests were conducted on each specimen, and the average value obtained was used in the analyses.

Stiffness and damping were estimated from the initial slope (modulus) and loop area of stress-strain responses, respectively. The effects of silica particles on the stiffness

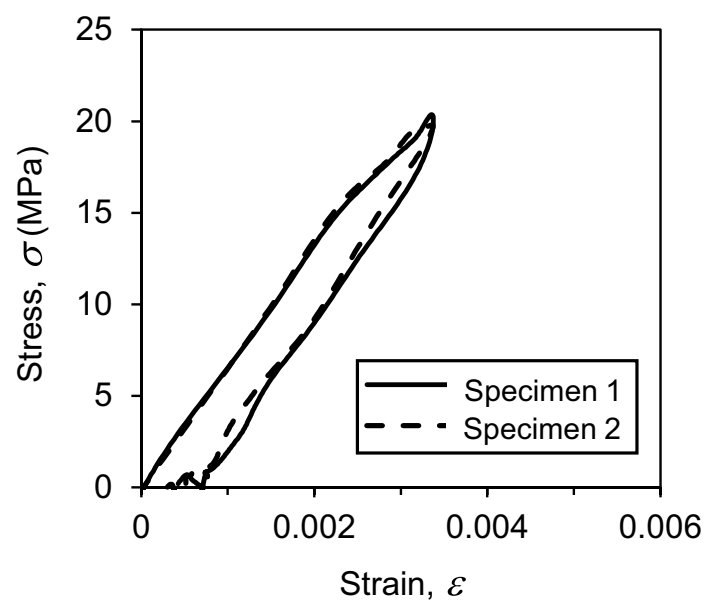

Fig. 7 Overlapped stress-strain responses of two epoxy/silica specimens indicate the reproducibility and consistency of experiments and damping of epoxy/silica adhesive were investigated for evaluation. Figures 8 and 9 show the stiffness and damping behaviors of epoxy/silica adhesive at silica contents of 2, 5, and $10 \mathrm{wt} \%$, as the silica composition ratio varies from pure microparticles $(\varphi=0 \%)$ to pure nanoparticles $(\varphi=100 \%)$.

The epoxy stiffness increased monotonically with increasing nanoparticle portion at a silica content of $2 \mathrm{wt} \%$ (Fig. 8). The influence became nonmonotonic with the increase of silica content to values of 5 and $10 \mathrm{wt} \%$. The stiffness of epoxy silica for any composition ratio increased with the increase of silica content up to $5 \mathrm{wt} \%$. However, the epoxy stiffness dropped as the silica particle content increased to $10 \mathrm{wt} \%$. At a silica content of $5 \mathrm{wt} \%$, it was observed that the stiffening effect of two-size silica was superior to that of

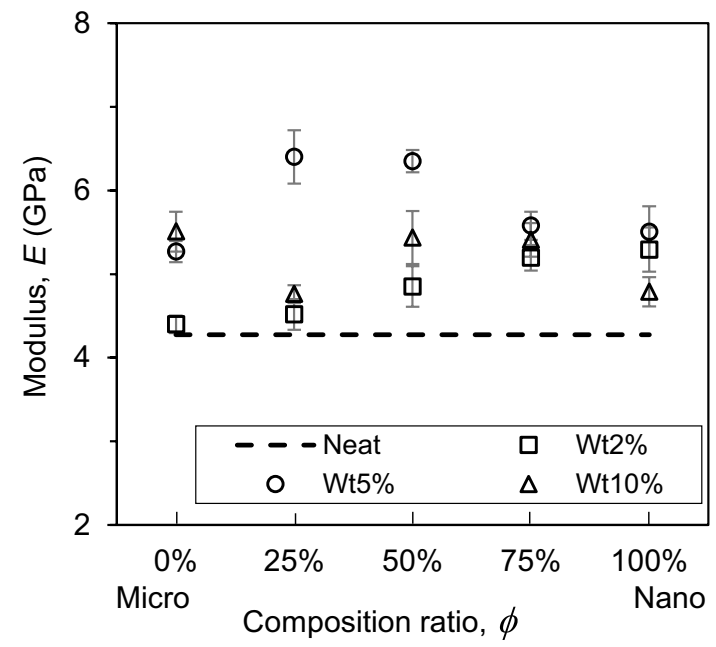

Fig. 8 Effects of silica content and composition ratio on the dynamic stiffness of epoxy

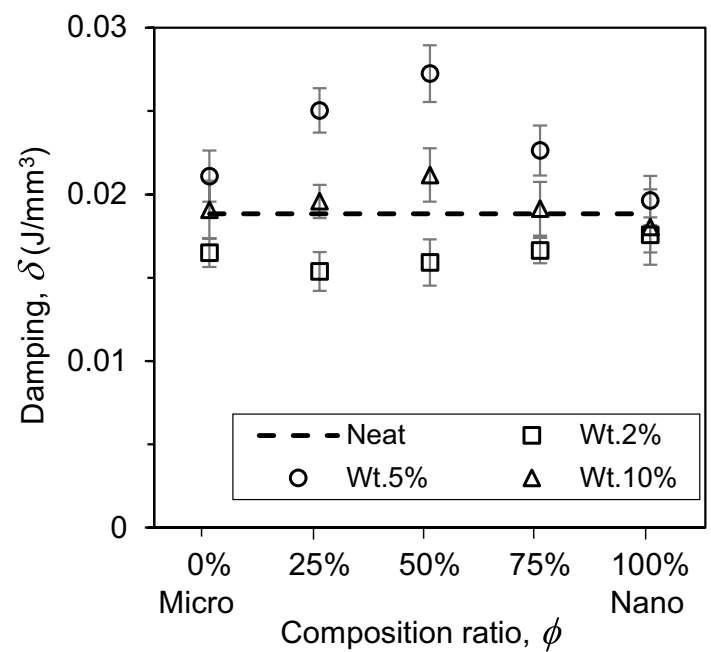

Fig. 9 Effects of silica content and composition ratio on the hysteretic damping of epoxy 
single-size silica. The greatest stiffening effect of two-size silica at $5 \mathrm{wt} \%$ was observed at the composition ratio of $25 \%$, where epoxy stiffness increased by $45 \%$ compared to that of neat epoxy.

The epoxy damping was nonmonotonically reduced by up to $14 \%$ with the two-size particles at a low silica content of $2 \mathrm{wt} \%$ (Fig. 9). Significant increases in epoxy damping occurred with the presence of two-size silica particles of any composition ratio as silica content increased to $5 \mathrm{wt} \%$. The highest epoxy damping was obtained at silica content of $5 \mathrm{wt} \%$ and a composition ratio of $50 \%$, where damping increased by $40 \%$ compared to that of neat epoxy. The damping reduced as silica content increased further to $10 \mathrm{wt} \%$.

Deviations were observed for both the estimated stiffness and damping. The main sources of deviations were variations in the specimens due to the mixing process. Initial slope fitting in stiffness estimation and fluctuating stress-strain loops near the end of the unloading stage also contributed to stiffness and damping deviations, respectively. Four specimens were used for each test condition to minimize deviation.

\section{Effects of Silica Micro-nanoparticles on Dynamic Stiffness}

It has been reported that dispersed silica particles form a matrix-filler interface area stiffer than the matrix and therefore stiffen and restrict deformation of epoxy adhesives [24, 25]. Epoxy stiffness increases with the interface area formed in the matrix, which is affected by the surface area of premixed silica particles and particle dispersion quality.

Figure 8 shows the stiffening effect of silica micro-nanoparticles at different weight fractions and composition ratios. A collaborative stiffening effect is only found on the epoxy filled with $5 \mathrm{wt} \%$ silica micro-nanoparticles. Micro-nanoparticles generate a stronger stiffening effect on the epoxy compared to those of either pure microparticles or pure nanoparticles. Figure 8 also reveals that $10 \mathrm{wt} \%$ silica particles generate weaker stiffening effects on epoxy adhesives even though it has a larger pre-mixed surface area, as shown in Fig. 10. This result shows the critical role of particle dispersion in transforming a pre-mixed surface area into an effective interface area. As a conventional mechanical mixer was used in the present work in the absence of any chemical treatment, dispersion quality was affected by particle size and weight fraction.

Figure 10 highlights the effects of silica weight fraction and composition ratio on the transformation of pre-mixed surface area into effective interface area that stiffens an epoxy adhesive. Large pre-mixed surface areas provided by 5 and $10 \mathrm{wt} \%$ silica with a high proportion of nanoparticles do not form large effective interface areas; therefore, these generate weak stiffening effects. However, micro-nanoparticles

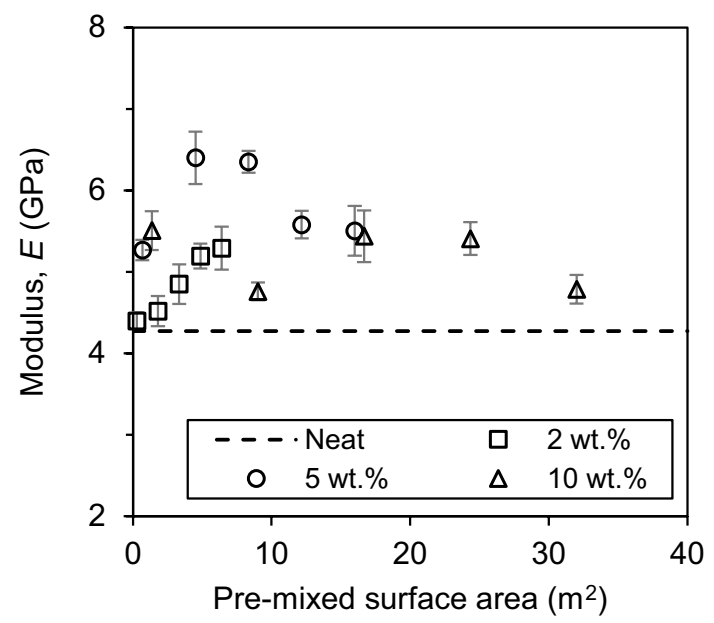

Fig. 10 Effects of silica content and pre-mixed surface area on the epoxy stiffness

at $5 \mathrm{wt} \%$ silica with a nanoparticle proportion of less than $75 \%$ effectively form large effective interface areas, thereby generating a strong stiffening effect. In the case of $2 \mathrm{wt} \%$ silica, micro-nanoparticles have only a weak effect on the formation of an effective interface area. Pure nanoparticles at $2 \mathrm{wt} \%$ silica are well dispersed, achieving a large effective interface area; therefore, these provide stronger stiffening effects than those of micro-nanoparticles. Good dispersion of low-content silica nanoparticles was observed, which is in agreement with previous works by Bondioli et al. [10], Zheng et al. [9], and Feli and Jalilian [8]. This result suggests that composing silica micro and nanoparticles in appropriate ratios improves their dispersion up to certain weight fraction when using only a conventional mechanical mixer.

The effectiveness of silica micro-nanoparticles in improving silica nanoparticle dispersion was verified by comparing the stiffness and strain response of the epoxy adhesive. Figure 11 shows the epoxy stiffness and strain responses of the epoxy adhesive used to estimate and compare the effective interface area. Based on the previously mentioned stiffening and strain restriction effects of the interface area on the epoxy adhesive, the epoxy with higher stiffness and smaller strain deformation contained a larger interface area (Fig. 11b, upper-left quadrant). Consequently, neat epoxy exhibited the lowest stiffness and largest strain deformation because it contained no interface area (Fig. 11b, lower-right quadrant). Epoxy filled with $5 \mathrm{wt} \%$ silica micro-nanoparticles exhibited the largest interface area and was the most sensitive to composition ratio variations; this was indicated by stronger stiffness-strain response with steeper slopes. The epoxy filled with $10 \mathrm{wt} \%$ silica micro-nanoparticles exhibited a smaller interface area and was less sensitive to composition ratio compared to the case of $5 \mathrm{wt} \%$. This result confirms that composing silica micro and nanoparticles 


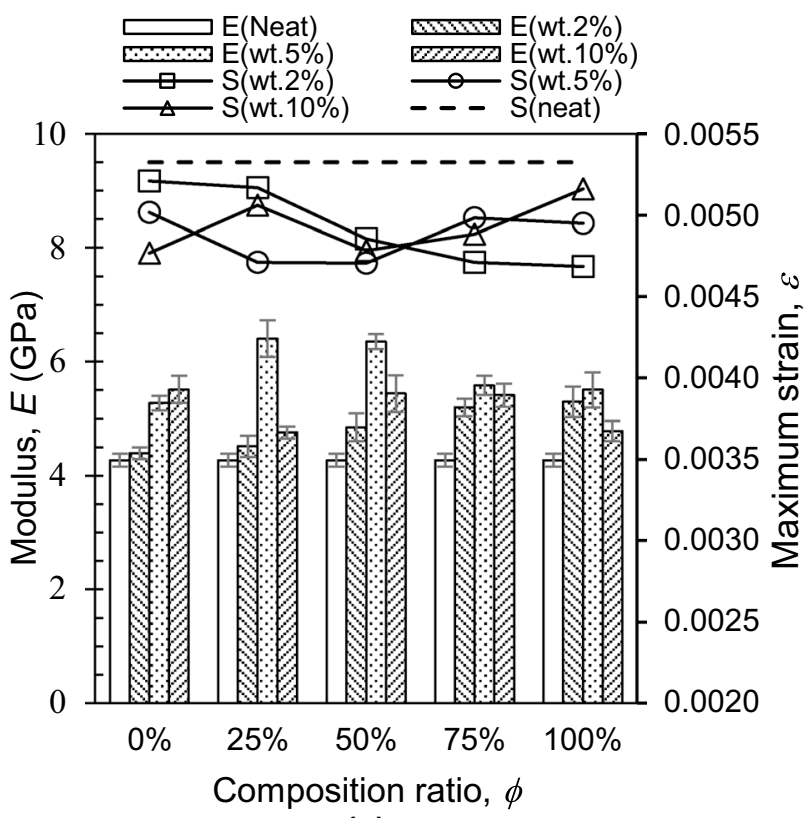

(a)

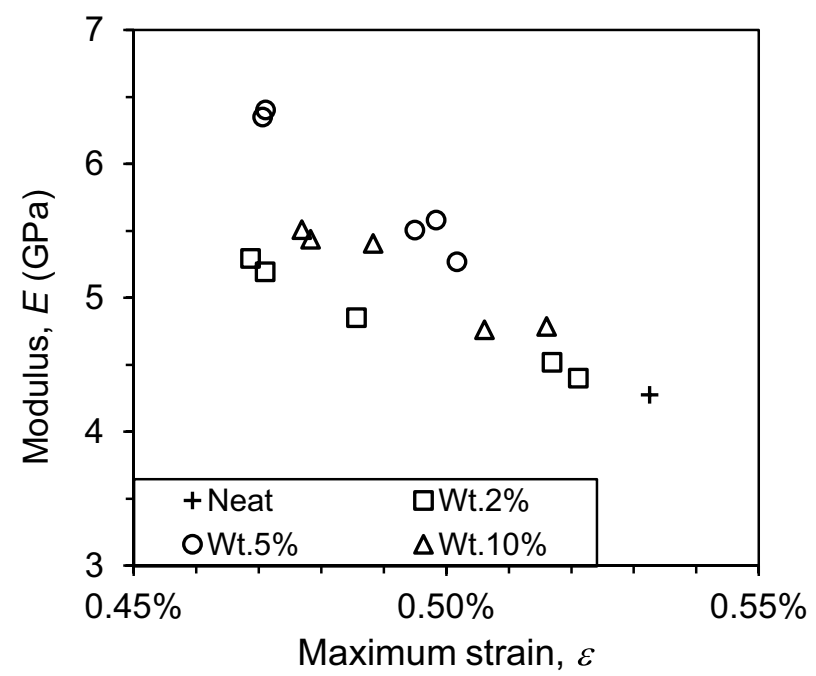

(b)

Fig. 11 The simultaneous effects of silica on the stiffeness and strain reducing (a), $E$ for stiffness and $S$ for strain. Proportional stiffnessstrain relation representing the effective interface area formed in the matrix (b)

effectively improves nanoparticle dispersion for silica content up to $5 \mathrm{wt} \%$ and generates a synergistic stiffening effect. It is worth noting that silica nanoparticle:microparticle compositions of $25: 75 \%$ or 50:50\% increase epoxy stiffness by $45 \%$ compared to that of neat epoxy.

In previous studies, Kwon et al. [14, 15], Dittanet and Pearson [16], and Shariati et al. [17], did not obtain a considerable cooperative stiffening effect of well-dispersed twosize silica particles. Kwon et al. and Shariati et al. mixed two-size silica particles, which have smaller size ratios
(1.56:0.24 $\mu \mathrm{m}$ and 17:65 $\mathrm{nm}$ in diameter, respectively) than that used in the present work ( $34 \mu \mathrm{m}: 17 \mathrm{~nm})$. Such smaller size ratios might be less effective in improving the interface area and generating collaborative stiffening effects. Olhero and Ferreira found that a larger size ratio of mixed silica particles resulted in better deagglomeration and thus better dispersion [26].

Dittanet and Pearson [16], however, combined silica particles of $42 \mu \mathrm{m}$ with 23,74 , and $170 \mathrm{~nm}$, but did not observe any considerable synergistic stiffening effect. It was confirmed by Dittanet and Pearson in the same paper that the stiffening effect of the interface area was neglected owing to weak matrix-filler adhesion.

Notably, Fig. 11b shows comparable ranges of strain deformations of epoxy filled with any silica weight fraction despite their different interface areas. Higher interface area should restrict deformation of the epoxy adhesive. This fact may indicate the existence of additional deformations induced by matrix-filler interactions. Particle debonding and stress concentration induce local matrix yielding or plastic deformation around the interface area. The mechanism of such deformations and its contribution to energy dissipation is discussed in the next subsection.

An alternative explanation for the additional strain deformation is a softer epoxy matrix due to poor cross-linking between the epoxy base and its hardener. Olhero and Ferreira reported that high nanoparticle content increases viscosity, and hence it is difficult to obtain homogeneous mixing [26]. Tarrio-Saavedra showed that higher nanoparticle content forms agglomerates that prevent perfect mixing of some part of the epoxy base and its hardener [27].

The results of this paper suggest that the poor dispersion performance of a conventional mechanical mixer can be improved by composing appropriate ratios of silica micronanoparticles. A synergistic stiffening effect is generated by the improved dispersion of silica nanoparticles up to a silica content of $5 \mathrm{wt} \%$. Beyond that silica content, mixed micronanoparticles with diameters of $34 \mu \mathrm{m}$ and $17 \mathrm{~nm}$ are less effective in improving dispersion. However, using two-size particles, which have different size ratios, may generate different results.

\section{The Synergistic Effect of Silica Micro-nanoparticles on Hysteretic Damping}

Owing to its inherent viscoelasticity, neat epoxy dissipates energy through internal friction during deformation, which can be estimated from the stress-strain loop area curve, as shown in Fig. 6.

The presence of high-modulus silica particles reduces epoxy deformation and thus reduces energy dissipation in the epoxy matrix. Therefore, the energy dissipation of epoxy with a silica content of $2 \mathrm{wt} \%$ is lower than that of neat 
epoxy, as shown in Fig. 12. Epoxy damping is reduced by $14 \%$ by the presence of $2 \mathrm{wt} \%$ silica particles. This small energy is likely to be dissipated through inter-particle slipping and friction in nanoparticle aggregates, as demonstrated by Yang et al. [28].

The energy dissipation values for epoxy with silica contents of 5 and $10 \mathrm{wt} \%$, however, are larger than that for neat epoxy. This increased energy dissipation indicates the existence of other energy dissipation mechanisms induced by matrix-filler interactions.

There are several mechanisms of energy dissipation induced by matrix-filler interactions suggested in previous literature $[16,29,30]$. Crack pining and crack bridging are dominant energy dissipation mechanisms for epoxy filled with pure silica microparticles. In epoxy filled only with

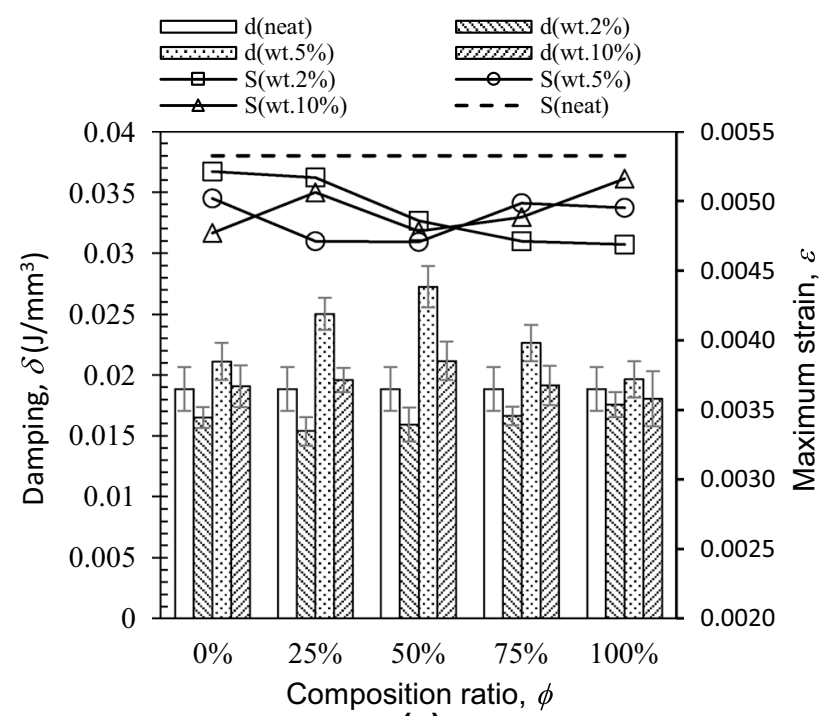

(a)

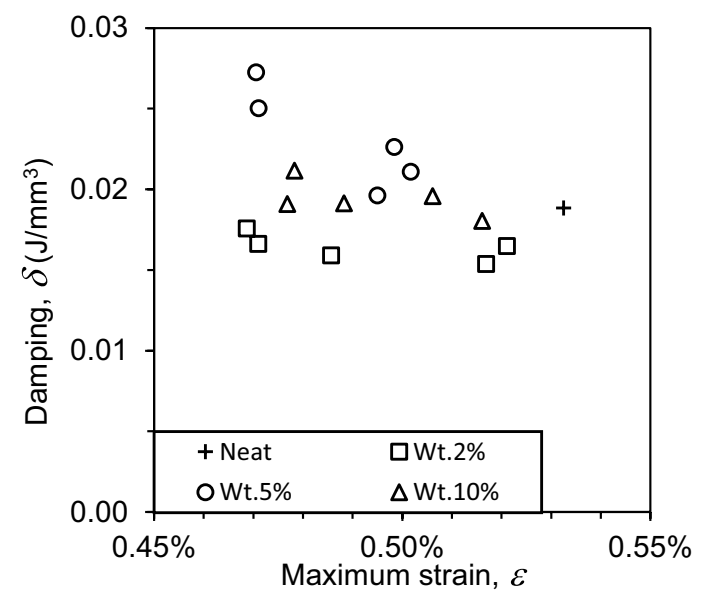

(b)

Fig. 12 The simultaneous effects of silica on the damping and strain reducing (a), $d$ for damping and $S$ for strain. Damping-strain relation of epoxy/silica and of neat epoxy (b) nanoparticles, energy is dissipated by local plastic deformation and matrix shear yielding induced by the nanoparticles debonding.

Epoxy filled with two-size silica particles exhibits superior energy dissipation to that of single-size silica particles owing to more complex matrix-filler interactions [16, 29]. This arises because of collegial mechanisms which boost epoxy energy dissipation such as nanoparticles and microparticles debonding followed by subsequent void formation and growth, crack deflection, and branching. In the present work, damping increases by $40 \%$ compared to that of neat epoxy at a silica content of $5 \mathrm{wt} \%$ composed of $50 \%$ microparticles and $50 \%$ nanoparticles.

The mechanisms mentioned above result in additional strain deformation in epoxy with 5 and $10 \mathrm{wt} \%$ silica, as confirmed in the previous subsection. There is evidence of yielding or damage occurrence: stress-strain responses deviate [31, 32] from linear slopes, as shown in Fig. 13.

There is also evidence of a disturbance in the specimen stress equilibrium during unloading, which may indicate damage in the epoxy/silica, as shown in Fig. 14. However, it is not clear whether this disturbance is caused by crack opening or by void expansion due to tension in the specimen. Damage also contributes to energy dissipation via cracks in the matrix and matrix/particle slipping in the interface area [28, 33].

Epoxy damping decreases as the silica content increases to $10 \mathrm{wt} \%$. The reduced matrix volume is one factor responsible for this lower damping performance. However, considering the small matrix volume reduction by the addition of $10 \mathrm{wt} \%$ silica particles (equal to $5.24 \mathrm{vol} \%$, Table 2), it is

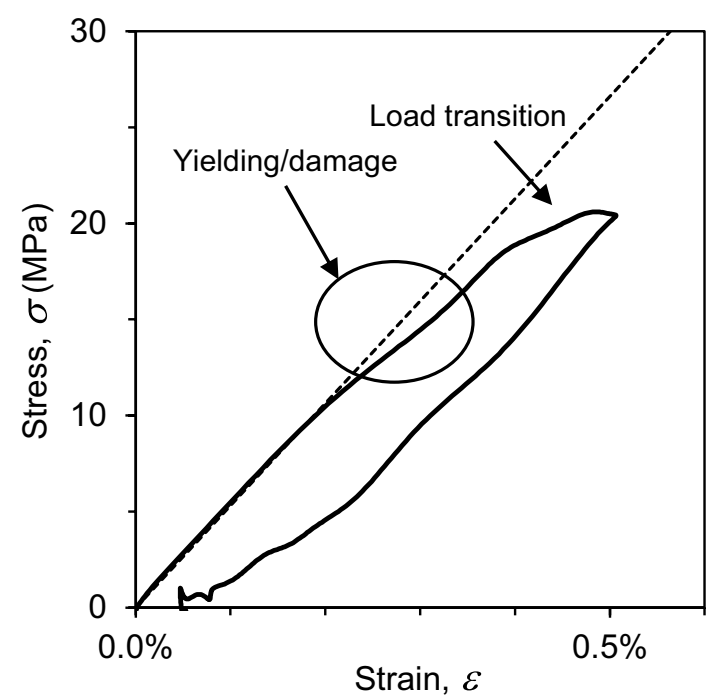

Fig. 13 Yield-like or damage behavior indicated by deviation of the stress-strain response from its elastic straight line 


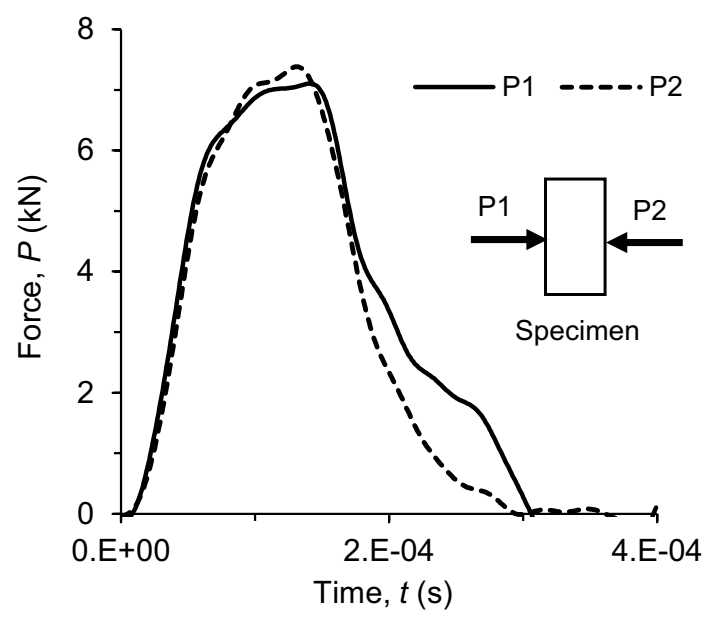

Fig. 14 Damage indication, which disturbed the stress equilibrium during unloading

inappropriate to ascribe poor damping performance to this matrix volume reduction alone.

As mentioned in the previous subsection, poor crosslinking between the epoxy base and its hardener is likely to occur. Some parts of the matrix that are poorly crosslinked do not exhibit viscoelastic properties such as hysteretic damping. Thus, the effective matrix volume, which enables energy dissipation, is reduced and results in poor damping performance.

The results in this study suggest that the damping performance of epoxy/silica is influenced by both inherent matrix properties and by matrix-filler interactions. Damping can be increased by adequate dispersal of an appropriate amount of two-size silica particles in an epoxy matrix, which generate cooperative matrix-filler interactions to dissipate energy.

\section{Optimum Composition Ratio and Weight Fraction of Silica Micro-nanoparticles}

Figure 15 summarizes the influences of silica micro-nanoparticles on dynamic stiffness and hysteretic damping of epoxy adhesives. There was no synergy effect at a low silica content of $2 \mathrm{wt} \%$ owing to the dominant effect of nanoparticles. Using pure silica nanoparticles was more beneficial than using micro-nanoparticles in that doing so efficiently stiffened the epoxy adhesive without reducing damping excessively.

A silica content of $5 \mathrm{wt} \%$ provided a wide range of stiffness and damping performance when varying the composition ratio; therefore, it furnishes a wide array of options for designing dynamic properties of epoxy adhesives appropriately for their applications. Moreover, both stiffness and damping can be increased by 45 and $40 \%$, respectively, at the optimum composition ratio of $\varphi=50 \%$.

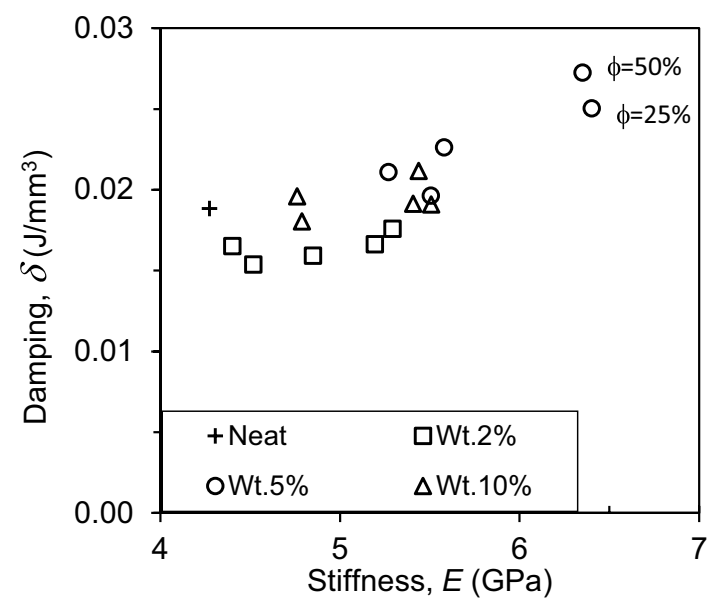

Fig. 15 Synergistic effects of mixed silica micro-nanoparticles on stiffness and damping performance

In contrast, a silica content of $10 \mathrm{wt} \%$ was less sensitive to the composition ratio and provided only weak performance in terms of stiffness and damping, which is a disadvantage in design applications. The best synergy effect at this silica content was obtained for higher microparticle proportions relative to nanoparticle proportions $(\varphi=25 \%)$.

\section{Conclusions}

The synergistic effects of silica micro-nanoparticles on the dynamic stiffness and damping of a bonded epoxy adhesive were investigated using split Hopkinson pressure bars. The effectiveness of selecting ratios of silica micro and nanoparticles for improving silica particle dispersion was discussed.

In the range of measurement, silica micro-nanoparticles of $34 \mu \mathrm{m}$ and $17 \mathrm{~nm}$ in average diameter, respectively, effectively improved nanoparticle dispersion, providing increased matrix-filler interaction through larger interface areas. Therefore, this composition generated synergistic stiffening and energy absorption effects in epoxy with a silica content of $5 \mathrm{wt} \%$. The synergistic stiffening effect was generated by the increased interface area due to the improved dispersion of the silica nanoparticles. The synergistic energy absorption effect was generated by yield and damage induced by complex interactions between the epoxy matrix and the silica micro-nanoparticles.

The effectiveness of silica micro-nanoparticles for improving the dispersion of silica particles is limited to 5 wt $\%$ when using a conventional mechanical mixer like the one used in this paper. However, there are possibilities for improving deagglomeration quality at higher silica contents using an appropriate composition ratio of two-size silica particles with a larger particle size ratio. 
The present paper provides experimental observations of epoxy dynamic stiffness and damping characteristics influenced by mixed silica micro-nanoparticles. By obtaining these characteristics, epoxy dynamic performance can be engineered for appropriate structural applications wherein epoxy is subjected to impact and vibration, such as in automobiles. In future work, another energy dissipation mechanism that avoids damage should be considered using hybrid filler materials such as a combination of soft and hard fillers.

Although yield or damage behavior, which contributes to energy dissipation, was identified and explained in this paper, a thorough investigation of matrix-filler interactions remains for future work to develop a better understanding of dynamic damping mechanisms.

Acknowledgements The authors would like to acknowledge the scholarship support from the Japanese Ministry of Education, Culture, Sports, Science, and Technology (MEXT) during this research study.

Open Access This article is distributed under the terms of the Creative Commons Attribution 4.0 International License (http://creativeco mmons.org/licenses/by/4.0/), which permits unrestricted use, distribution, and reproduction in any medium, provided you give appropriate credit to the original author(s) and the source, provide a link to the Creative Commons license, and indicate if changes were made.

\section{References}

1. Dittanet P, Pearson RA (2012) Effect of silica nanoparticle size on toughening mechanisms of filled epoxy. Polymer 53(9):1890-1905

2. He P, Huang M, Yu B, Sprenger S, Yang J (2016) Effects of nanosilica contents on the properties of epoxy nanocomposites and Ti-epoxy assembles. Compos Sci Technol 129:46-52

3. Rostamiyan Y, Fereidoon A, Ghasemi Ghalebahman A, Hamed Mashhadzadeh A, Salmankhani A (2015) Experimental study and optimization of damping properties of epoxy-based nanocomposite: effect of using nanosilica and high-impact polystyrene by mixture design approach. Mater Des (1980-2015) 65:1236-1244

4. Zamanian M, Mortezaei M, Salehnia B, Jam JE (2013) Fracture toughness of epoxy polymer modified with nanosilica particles: particle size effect. Eng Fract Mech 97:193-206

5. Adachi T, Imai Y, Higuchi M (2011) Fracture Energy of Nano-and micro-silica particle-filled epoxy composites. Int J Theor Appl Multiscale Mech 2(1):82-94

6. Conradi M, Zorko M, Kocijan A, Verpoest I (2013) Mechanical properties of epoxy composites reinforced with a low volume fraction of nanosilica fillers. Mater Chem Phys 137(3):910-915

7. Islam MS, Masoodi R, Rostami H, Islam MS, Masoodi R, Rostami $H$ (2013) The effect of nanoparticles percentage on mechanical behavior of silica-epoxy nanocomposites. J Nanosci. https://doi. org/10.1155/2013/275037

8. Feli S, Jalilian MM (2016) Experimental and optimization of mechanical properties of epoxy/nanosilica and hybrid epoxy/ fiberglass/nanosilica composites. J Compos Mater 50:3891

9. Zheng Y, Chonung K, Wang G, Wei P, Jiang P (2008) Epoxy/ nano-silica composites: curing kinetics, glass transition temperatures, dielectric, and thermal-mechanical performances. J Appl Polym Sci 111:917
10. Bondioli F, Cannillo V, Fabbri E, Messori M (2005) Epoxy-silica nanocomposites: preparation, experimental characterization, and modeling. J Appl Polym Sci 97(6):2382-2386

11. Greenwood R, Luckham PF, Gregory T (1997) The effect of diameter ratio and volume ratio on the viscosity of bimodal suspensions of polymer latices. J Colloid Interface Sci 191(1):11-21

12. Greenwood R, Luckham PF, Gregory T (1998) Minimising the viscosity of concentrated dispersions by using bimodal particle size distributions. Colloids Surf Physicochem Eng Asp 144(1):139-147

13. Dames B, Morrison BR, Willenbacher N (2001) An empirical model predicting the viscosity of highly concentrated, bimodal dispersions with colloidal interactions. Rheol Acta 40(5):434-440

14. Kwon SC, Adachi T, Araki W, Yamaji A (2005) Effect of particle size on fracture toughness of spherical-silica particle filled epoxy composites. Key Eng Mater 297-300:207-212

15. Kwon S-C, Adachi T, Araki W, Yamaji A (2008) Effect of composing particles of two sizes on mechanical properties of spherical silica-particulate-reinforced epoxy composites. Composite B 39(4):740-746

16. Dittanet P, Pearson RA (2013) Effect of bimodal particle size distributions on the toughening mechanisms in silica nanoparticle filled epoxy resin. Polymer 54(7):1832-1845

17. Shariati M, Farzi GA, Dadrasi A, Amiri M, Meybodi RR (2015) An experimental study on toughening mechanisms of fillers in epoxy/silica nanocomposites. Int $\mathbf{J}$ Nanosci Nanotechnol 11(3): 193-199

18. Miao Y-G, Liu H-Y, Suo T, Mai Y-W, Xie F-Q, Li Y-L (2016) Effects of strain rate on mechanical properties of nanosilica/epoxy. Composite B 96:119-124

19. Tian $Y$ et al (2016) High strain rate compression of epoxy based nanocomposites. Composite A 90:62

20. Yohanes, Sekiguchi Y (2017) Effects of mixed micro and nano silica particles on the dynamic compressive performances of epoxy adhesive. Appl Adhes Sci 5(1):3

21. Song B, Chen W (2004) Loading and unloading split hopkinson pressure bar pulse-shaping techniques for dynamic hysteretic loops. Exp Mech 44(6):622-627

22. Cloete TJ, Paul G, Ismail EB (2014) Hopkinson bar techniques for the intermediate strain rate testing of bovine cortical bone. Philos Trans R Soc Math Phys Eng Sci 372(2015):20130210-20130210

23. Chen W, Song B, Frew DJ, Forrestal MJ (2003) Dynamic small strain measurements of a metal specimen with a split Hopkinson pressure bar. Exp Mech 43(1):20-23

24. Wang H, Bai Y, Liu S, Wu J, Wong CP (2002) Combined effects of silica filler and its interface in epoxy resin. Acta Mater 50(17):4369-4377

25. Hua Y, Gu L, Premaraj S, Zhang X (2015) Role of interphase in the mechanical behavior of silica/epoxy resin nanocomposites. Materials 8(6):3519-3531

26. Olhero S, Ferreira JM (2004) Influence of particle size distribution on rheology and particle packing of silica-based suspensions. Powder Technol 139(1):69-75

27. Tarrio-Saavedra $\mathbf{J}$ (2010) Controversial effects of fumed silica on the curing and thermomechanical properties of epoxy composites. Express Polym Lett 4(6):382-395

28. Yang R, Song Y, Zheng Q (2017) Payne effect of silica-filled styrene-butadiene rubber. Polymer 116:304-313

29. Keivani M, Khamesinia A, Bagheri R, Kouchakzadeh MA, Abadyan M (2015) Study of synergistic toughening in a bimodal epoxy nanocomposite. J Reinf Plast Compos 34(4):281-292

30. Sun L, Gibson RF, Gordaninejad F, Suhr J (2009) Energy absorption capability of nanocomposites: a review. Compos Sci Technol 69(14):2392-2409

31. Kara A, Tasdemirci A, Guden M (2013) Modeling quasi-static and high strain rate deformation and failure behavior of a $( \pm 45)$ 
symmetric E-glass/polyester composite under compressive loading. Mater Des 49:566-574

32. Song B, Chen W, Yanagita T, Frew DJ (2005) Confinement effects on the dynamic compressive properties of an epoxy syntactic foam. Compos Struct 67(3):279-287
33. Kroisová D (2010) An internal damping in epoxy composite systems. In: Proceeding of ISMA international conference on noise and vibration engineering, Belgium 